\title{
TIME ASYMPTOTICS OF THE SCHRÖDINGER WAVE FUNCTION IN TIME-PERIODIC POTENTIALS
}

\author{
O. COSTIN, R. D. COSTIN, AND J. L. LEBOWITZ ${ }^{1}$ \\ DEPARTMENT OF MATHEMATICS, RUTGERS UNIVERSITY
}

Dedicated to Elliott Lieb on the occasion of his 70th birthday

\begin{abstract}
We study the transition to the continuum of an initially bound quantum particle in $\mathbb{R}^{d}, d=1,2,3$, subjected, for $t \geq 0$, to a time periodic forcing of arbitrary magnitude. The analysis is carried out for compactly supported potentials, satisfying certain auxiliary conditions. It provides complete analytic information on the time Laplace transform of the wave function. From this, comprehensive time asymptotic properties (Borel summable transseries) follow.

We obtain in particular a criterion for whether the wave function gets fully delocalized (complete ionization). This criterion shows that complete ionization is generic and provides a convenient test for particular cases. When satisfied it implies absence of discrete spectrum and resonances of the associated Floquet operator. As an illustration we show that the parametric harmonic perturbation of a potential chosen to be any nonzero multiple of the characteristic function of a measurable compact set has this property.
\end{abstract}

Keywords: Ionization, delocalization, resonances, Floquet theory, Borel summability.

\section{INTRODUCTION}

We consider the non-relativistic Schrödinger equation for the wave function $\psi(x, t), x \in \mathbb{R}^{d}$

$$
i \frac{\partial \psi}{\partial t}=(-\Delta+V(x)+\Omega(x, t)) \psi
$$

where $\Omega(x, t)$ is a time-periodic external potential (not necessarily small):

$$
\Omega(x, t)=\Omega(x, t+2 \pi / \omega), \omega>0
$$

We take $V$ and $\Omega$ real-valued and so that

$$
V \in L^{\infty}\left(\mathbb{R}^{d}\right), \quad \Omega \in L^{\infty}\left(\mathbb{R}^{d} \times[0,2 \pi / \omega]\right)
$$

with $\Omega \not \equiv 0$ satisfying

$$
\Omega(x, t)=\sum_{j \in \mathbb{Z}} \Omega_{j}(x) e^{i j \omega t}, \quad \Omega_{j}(x)=\overline{\Omega_{-j}(x)} ; \quad \sup _{j, x}\left|\Omega_{j}(x)\right| j^{2}<\infty
$$

We set, without loss of generality,

$$
\Omega_{0}(x)=0
$$

\footnotetext{
${ }^{1}$ Also Department of Physics.
} 
We are interested in the behavior of solutions $\psi(x, t)$ for large $t$ when

$$
\psi(x, 0)=: \psi_{0}(x) \in L^{2}\left(\mathbb{R}^{d}\right), \quad \int_{\mathbb{R}^{d}}\left|\psi_{0}\right|^{2} d x=1
$$

and $\psi_{0}$ is sufficiently regular (we assume it of class $C^{4}$ ). Of particular interest is the survival probability for the particle in a ball $B$ in $\mathbb{R}^{d}, \int_{x \in B}|\psi|^{2} d x:=\mathcal{P}_{B}(t)$. If $\mathcal{P}_{B}(t)$ approaches zero as $t \rightarrow \infty$ for all $B$, then we say that the particle escapes to infinity and complete ionization occurs.

While many results in the paper only require (1.3) (1.4) plus sufficient algebraic decay of $\Omega$ and $V$ for large $|x|$, some specific results later in the paper, particularly detailed analytic information, require that $V$ and $\Omega$ are compactly supported,

$$
\operatorname{supp}(V) \cup \operatorname{supp}(\Omega(\cdot, t)) \subset D
$$

and others require the same also for $\psi_{0}$ in (1.6)

$$
\operatorname{supp}\left(\psi_{0}\right) \cup \operatorname{supp}(V) \cup \operatorname{supp}(\Omega(\cdot, t)) \subset D
$$

with

$$
D \subset \mathbb{R}^{d} \text { compact, } \mathbb{R}^{d} \backslash D \text { connected, meas }(\partial D)=0
$$

The rest of the paper will therefore be written in the context of this setting.

1.1. Nature of the results. Under the assumptions (1.7b) $\psi(x, t)$ is obtained for large $t$ as a convergent combination of exponentials and Borel summable power series in $t^{-1 / 2}$.

If an additional assumption (connected to the absence of discrete spectrum of the Floquet operator) is satisfied, the long time expression of $\psi$ contains only decaying terms, cf. Theorem 12 in $\S 3.8$ i.e. we get complete ionization. ${ }^{1}$

We find in Proposition 14 a convenient sufficient condition for complete ionization, and show that it is satisfied by a nonperturbative example ${ }^{2}$

$$
V(x)=V_{D} \chi_{D}(x) ; \quad \Omega(x, t)=2 \Omega_{D} \chi_{D}(x) \sin \omega t
$$

where $\chi_{D}$ is the characteristic function of $D$ in $d=1,2,3$ and $V_{D}$ and $\Omega_{D}$ are arbitrary nonzero constants.

We previously obtained similar results for more general potentials in $d=1$ and radially symmetric ones in $d=2,3$. See [7, 8, 9, 10, 11] and [6] where there is a review of our previous work on this problem.

1.2. Strategy of the approach. The key steps of our approach are outlined in 3.4 The method we use is based on a study of the analytic properties of $\hat{\psi}$, the Laplace transform of $\psi$. The type and position of the singularities of $\hat{\psi}$, given in Theorem 7 and Lemma 8 provide information about the time behavior of $\psi$; the former are obtained from an appropriate equation to which the Fredholm alternative approach applies.

\footnotetext{
${ }^{1}$ While explicit information on long time behavior requires $\psi_{0}$ to be localized, decay for more general $\psi_{0} \in L^{2}$ is then an immediate consequence of the unitarity of Schrödinger evolution.

${ }^{2}$ The same results hold if a bounded time-independent potential, not necessarily constant, with compact support disjoint from $D$ is added to $V$, see Remark 25
} 


\section{LAplace transform, LinK With Floquet TheORY}

Exisence of a strongly differentiable unitary propagator for (1.1) (see 23] v.2, Theorem X.71) implies that for $\psi_{0} \in L^{2}\left(\mathbb{R}^{d}\right)$, the Laplace transform

$$
\hat{\psi}(\cdot, p):=\int_{0}^{\infty} \psi(\cdot, t) e^{-p t} d t
$$

exists for $\Re(p)>0$. It satisfies the equation

$$
(-\Delta+V(x)-i p) \hat{\psi}(x, p)=-i \psi_{0}-\sum_{j \in \mathbb{Z}} \Omega_{j}(x) \hat{\psi}(x, p-i j \omega)
$$

and the map $p \rightarrow \psi(\cdot, p)$ is $L^{2}$ valued analytic in the right half plane

$$
p \in \mathbb{H}=\{z: \Re(z)>0\}
$$

Clearly, equation (2.2) couples $\hat{\psi}\left(x, p_{1}\right)$ with $\hat{\psi}\left(x, p_{2}\right)$ iff $\left(p_{1}-p_{2}\right) \in i \omega \mathbb{Z}$. Setting

$$
p=i(\sigma+n \omega) \text { with } \Re \sigma \in[0, \omega)
$$

(sometimes it will be technically helpful to relax this restriction on $\sigma$ ) we define $y_{n}^{[1]}(x ; \sigma)=\hat{\psi}(x, i(\sigma+n \omega))$. Eq (2.2) now becomes a differential-difference system

$$
(-\Delta+V+\sigma+n \omega) y_{n}^{[1]}=-i \psi_{0}-\sum_{j \in \mathbb{Z}} \Omega_{j}(x)\left(S^{-j} y^{[1]}\right)_{n}
$$

where the shift operator $S$ is given by

$$
(S y)_{n}=y_{n+1}
$$

2.1. Connection with Floquet theory. The solution of (1.1) with time periodic $\Omega$ is of course the subject of Floquet theory (see [2] and [16]-23]) and therefore our analysis connects to it in a number of ways. Let $K$ be the quasi-energy operator in Floquet theory

$$
(K u)(x, \theta)=\left(-i \frac{\partial}{\partial \theta}-\Delta+V(x)+\Omega(x, \theta)\right) u(x, \theta) ; x \in \mathbb{R}^{d}, \theta \in S_{2 \pi / \omega}^{1}
$$

Then, letting

$$
u(x, \theta ; \sigma)=\sum_{n \in \mathbb{Z}} y_{n}^{[1]}(x ; \sigma) e^{i n \omega \theta}
$$

be the solution of the eigenvalue equation

$$
K u=-\sigma u
$$

we get an equation for the $y_{n}^{[1]}$ which is identical to the homogeneous part of equation $(2.5)^{3}$. Solutions of (2.9) with $u \in L^{2}\left(\mathbb{R}^{d} \times S_{2 \pi / \omega}^{1}\right)$ correspond to eigenfunctions of $K$.

Remark 1. If $u$ is an eigenfunction of $K$ corresponding to the eigenvalue $-\sigma$, then $u e^{-i j \omega \theta}$ is an eigenfunction with eigenvalue $-\sigma+j \omega$. For this reason it is enough to restrict $\sigma$ to the strip given in 2.4.

\footnotetext{
${ }^{3}$ The functional spaces are different. Proposition 18 clarifies this question.
} 
Complete ionization clearly requires the absence of a discrete spectrum of (2.9) (Otherwise, if $u(x, \theta)$ is an eigenfunction of $K$, then $e^{i \sigma t} u(x, t)$ would be a space localized solution of the Schrödinger equation.) In a recent work [16], Galtbayar, Jensen and Yajima proved that the opposite is also true. They obtained asymptotic series in $t^{-1 / 2}$ for the projection of the wave function $\psi(x, t)$ on the space orthogonal to the discrete spectrum of $K$.

Our approach via Laplace transform is different from that of 16 . For the corresponding time evolution our results are stronger than those obtained in [16] but apply to the more restrictive classes of $V$ and $\Omega$ satisfying $1.7 \mathrm{~b}$ in $d=1,2,3$. We show that the time behavior of $\psi(x, t)$ is given by a Borel summable transseries containing both power law decay and exponential terms. For potentials satisfying our condition (1.8) we show that $K$ has no discrete spectrum or resonances and all the exponentials are decaying.

More details on the connection between our work, Floquet theory and 16] are given in $\$ 5$

\section{Integral EQUATION, COMPACTNESS AND ANALYTICITY}

3.1. Laplace space equation. To simplify contour deformation, we first improve the decay of $\hat{\psi}$ for large $p$, by pulling the first two terms in the asymptotic behavior for large $p$ from $\hat{\psi}$. Let $\delta_{i j}=1$ if $i=j$, and 0 otherwise, $\delta_{i j}^{c}=1-\delta_{i j}$ and define the operator $\mathfrak{N}$ by

$$
(\mathfrak{N} f)_{n}(x)=(-\Delta+V) \frac{\delta_{n 0}^{c} f_{n}(x)}{\sigma+n \omega}-\delta_{n 0} f_{n, x}+\sum_{k \neq n} \Omega_{n-k}(x) \frac{\delta_{k 0}^{c} f_{k}(x)}{\sigma+k \omega}
$$

3.2. Green function representation. To pass to an integral form of the system of equations (3.12) we apply to them the Green function of $(-\Delta+\sigma+n \omega)$, given by

$$
\left(\mathfrak{g}_{n} f\right)(x)=\int G\left(\kappa_{n}\left(x-x^{\prime}\right)\right) f\left(x^{\prime}\right) d x^{\prime}
$$

with

$$
\kappa_{n}=\sqrt{-i p}=\sqrt{\sigma+n \omega} \text { (when } p \in \mathbb{H}, \kappa_{n} \text { is in the fourth quadrant) }
$$

and

$$
G\left(\kappa_{n} x\right)=\left\{\begin{array}{cc}
\frac{1}{2} \kappa_{n}^{-1} e^{-\kappa_{n}|x|} & d=1 \\
\frac{1}{2 \pi} K_{0}\left(\kappa_{n}|x|\right) & d=2 \\
\frac{1}{4 \pi}|x|^{-1} e^{-\kappa_{n}|x|} & d=3
\end{array}\right.
$$

(see [23]) where $K_{0}$ is the modified Bessel function of second kind,

$$
K_{0}(x)=\int_{0}^{\infty} e^{-x \cosh t} d t=e^{-x} \int_{0}^{\infty} \frac{e^{-x s}}{\sqrt{s(s+2)}} d s
$$

Note that, in the setting (1.7a), for $f$ supported in $D$ we have

$$
\left(\mathfrak{g}_{n} f\right)(x)=\int_{D} G\left(\kappa_{n}\left(x-x^{\prime}\right)\right) f\left(x^{\prime}\right) d x^{\prime}
$$


Eq. (3.1) in integral form becomes

$$
y_{n}^{[1]}=-i \mathfrak{g}_{n} \psi_{0}+\left(\mathfrak{C} y^{[1]}\right)_{n}
$$

where

$$
y^{[1]}=\left(y_{n}^{[1]}\right)_{n \in \mathbb{Z}} \text { and }\left(\mathfrak{C} y^{[1]}\right)_{n}=-\mathfrak{g}_{n}\left[V y_{n}^{[1]}+\sum_{j \in \mathbb{Z}} \Omega_{j}(x)\left(S^{-j} y^{[1]}\right)_{n}\right]
$$

To ensure better decay with respect to $n$ we further substitute in 3.8

$$
y_{n}^{[1]}=-i \mathfrak{g}_{n} \psi_{0}-i \psi_{1, n}+y_{n}
$$

where $\left(\psi_{1, n}\right)_{n \in \mathbb{Z}}=: \psi_{1}$ and

$$
\psi_{1}=\mathfrak{C}\left[\left(\mathfrak{g}_{n} \psi_{0}\right)_{n \in \mathbb{Z}}\right]
$$

Then $y$ satisfies

$$
y=w+\mathfrak{C}(\sigma) y
$$

(We write $\mathfrak{C}$ for $\mathfrak{C}(\sigma)$ when the dependence on $\sigma$ need not be stressed.) In differential form (3.11) reads

$$
(-\Delta+\sigma+n \omega) y_{n}=i \psi_{2, n}-V y_{n}-\sum_{j \in \mathbb{Z}} \Omega_{j}(x)\left(S^{-j} y\right)_{n}
$$

3.3. The Hilbert space. To analyze the properties of (3.11) we use the Hilbert space

$$
\mathcal{H}=l_{\gamma}^{2}\left(L^{2}(B)\right)
$$

where $B$ is an arbitrary ball (containing $D$ ) defined as the space of sequences $\left\{y_{n}\right\}_{n \in \mathbb{Z}}, y_{n} \in L^{2}(B)$ with

$$
\|y\|_{\mathcal{H}}^{2}=\sum_{n \in \mathbb{Z}}|n|^{\gamma}\left\|y_{n}\right\|_{L^{2}(B)}^{2}<\infty
$$

and adequate $\gamma$; we take for definiteness $\gamma=3 / 2$; larger $\gamma$ can be taken if one assumes more differentiability than (1.4) implies. (Note that $\mathcal{H}$ is different from the Hilbert space $L^{2}\left(L^{2}\left(\mathbb{R}^{d}\right)\right)$ used in Floquet theory. See also $₫$ )

3.4. Strategy of the approach, continued. As mentioned, unitarity of the evolution shows that $\hat{\psi}(\cdot, p) \in L^{2}\left(\mathbb{R}^{d}\right)$ if $\Re(p)>0$. In the integral form (3.11), whose solutions are in $\mathcal{H}$ when $\Re(p)>0$, the operator is, under our assumptions, compact. The solution of this equation is shown to be unique in $\mathcal{H}$ for large enough $\Re(p)$ by the contractivity of the integral operator. Uniqueness and analyticity of the solution for $p$ in the right half plane $\mathbb{H}$ follow by an application of the analytic version of the Fredholm alternative [23]. We then show that the solution and thus $\hat{\psi}$ are analytic with respect to a uniformizing variable, in appropriately chosen domains containing parts of the imaginary axis. The contour of the inverse Laplace transform, $\tau \rightarrow c+i \tau ; \tau \in \mathbb{R}, c>0$, can then be deformed to $i \mathbb{R}$ (the boundary of $\mathbb{H})$ where $\hat{\psi}$ is analytic except for a discrete set of square root branch points. The large time behavior of $\psi$ follows. 
3.5. Compactness. In $d=1$, we further transform the equation, see 88.3 in Appendix to improve the regularity of the operator at $n=0$ and $\sigma=0$.

Lemma 2. Under the assumptions 1.7a), $w \in \mathcal{H}$ and $\mathfrak{C}$ is a compact operator on $\mathcal{H}$.

To show that $w \in \mathcal{H}$ we use the fact that the operators $\mathfrak{g}_{n}$ satisfy (see Appendix A of [1], and also $\$ 7.1$.

$$
\sup _{n \in \mathbb{Z}}(1+|n|)^{1 / 2}\left\|\mathfrak{g}_{n}\right\|_{L^{2}(\mathcal{D})}<\infty
$$

Then

$$
\sup _{n \in \mathbb{Z}}(1+|n|)^{1 / 2}\left\|\left(\mathfrak{g}_{n} \psi_{0}\right)_{n}\right\|<\infty \text { implying } \sup _{n \in \mathbb{Z}}(1+|n|)\left\|\psi_{1, n}\right\|_{L^{2}(\mathcal{D})}<\infty
$$

In view of (1.4) we also have

$$
\sup _{n \in \mathbb{Z}}(1+|n|)\left\|V \psi_{1, n}+\sum_{j \in \mathbb{Z}} \Omega_{j}(x)\left(S^{-j} y\right)_{n}\right\|_{L^{2}(\mathcal{D})}<\infty
$$

implying

$$
\sup _{n \in \mathbb{Z}}\left(1+|n|^{3 / 2}\right)\left\|w_{n}\right\|_{L^{2}(\mathcal{D})}<\infty
$$

It is not difficult to check that $\mathfrak{g}_{n}$ is compact on $L^{2}(B)$ for each $n$; it is more delicate to show compactness of $\mathfrak{C}$; both properties are proved in 9

\subsection{Uniqueness.}

Lemma 3. For large enough $-\Im \sigma$, eq. 3.11) has a unique solution in $\mathcal{H}$.

The proof is given in Appendix B

\subsection{Analytic structure of $\hat{\psi}$.}

Remark 4. It is convenient to introduce the uniformizing variable $\sigma=u^{2}$; with the natural branch of the square root, $u$ is in the fourth quadrant when $\sigma$ is in the lower half plane. In this variable, we write $\kappa_{0}=u$ and $\kappa_{n}=\sqrt{n \omega+u^{2}}$ for $n \neq 0$.

Proposition 5. In the setting (1.7a) the operator $\mathfrak{C}(\sigma)$ is analytic in $u$ in the region $S_{\omega}=\left\{u:\left|\Re u^{2}\right|<\omega\right\}$ hence in $\sqrt{\sigma}$ in the strip (see Remark 1)

$$
\{\sigma: \Re(\sigma) \in(-\omega, \omega)\}
$$

Additionally, $\mathfrak{C}(\sigma)$ is analytic in $\sigma$ at any $\sigma \neq 0$.

Proof. In terms of $u$ we define $\kappa_{0}=u, \kappa_{n}=\sqrt{u^{2}+n \omega}$ and then $\kappa_{n}$ is analytic in $u$ in the simply connected region $S_{\omega}$ for any $n \in \mathbb{Z}$. Since in the setting (1.7a) the integral (3.6) is over a compact set, $D, \mathfrak{g}_{n}$ is also analytic for any $n \in \mathbb{Z}$. Analyticity of $\mathfrak{C}$ follows from the fact that

$$
\mathfrak{C}=\lim _{N \rightarrow \infty} \mathfrak{C}_{N}
$$

with

$$
\left(\mathfrak{C}_{N} y\right)_{n}:=\left\{\begin{array}{cc}
(\mathfrak{C} y)_{n} ; & |n| \leq N \\
0 & \text { otherwise }
\end{array}\right.
$$

and convergence is uniform in $u$ on compact subsets of $S_{\omega}$. This is shown in Lemma 27 
Proposition 6. There exists a unique solution y to 3.11) and it has the same analyticity properties as $\mathfrak{C}$ if

$$
\text { (A) } \text { For } \Im \sigma \leq 0 \quad(v=\mathfrak{C} v, v \in \mathcal{H}) \Rightarrow v=0
$$

Proof. This is nothing more than the analytic Fredholm alternative (see e.g. 23] Vol 1, Theorem VI.14, pp. 201).

This formulation is convenient in determining the analytic properties of $y$ with respect to $\sigma$, instrumental for the Borel summability results stated in Proposition 10 .

Theorem 7. If (A) and (1.7b) hold, then:

(i) the solution $y$ of 3.11) is meromorphic in $u$ in the disk $\{u:|u|<\sqrt{\omega}\}$, see Remark 4. analytic at $u=0$ and in the fourth quadrant of $S_{\sqrt{\omega}}$. Furthermore, $y$ is analytic in $\sigma$ at any $\sigma_{0} \neq 0$.

(ii) $\hat{\psi}$ is analytic in $p$ in a cut neighborhood of $i \mathbb{R},\{p: \Re(p)>-\epsilon\}$ with cuts toward $-\infty$ at in $\omega, n \in \mathbb{Z}$. Furthermore, in a neighborhood of in $\omega$, see [2.4), we have $\hat{\psi}(p)=A_{n}(p)+B_{n}(p) \sqrt{\sigma}$ where $A_{n}$ and $B_{n}$ are analytic at $i n \omega$ and, for some $\epsilon<\sqrt{\omega}$ and $|u| \leq \epsilon$ we have

$$
\sup _{n \in \mathbb{Z},|u|<\epsilon} n^{\gamma}\left(\left\|A_{n}(p)\right\|_{L^{2}(B)}+\left\|B_{n}(p)\right\|_{L^{2}(B)}\right)<\infty
$$

Proof. (i) follows from Propositions 5 and 6 and from the link between $\hat{\psi}$ and $y_{n}$.

(ii) The functions $A_{n}$ and $B_{n}$, are simply the even and odd part respectively of the analytic function $y_{n}(x ; u)$. The estimate follows from the fact that $y(x ; u) \pm$ $y(x ;-u) \in \mathcal{H}$ is analytic in $u$ for $|u|<\epsilon$.

In Proposition 10] and Theorem [12 below we use the following result.

Lemma 8. In the setting (1.7a), if $\hat{\psi}$ has a pole at $\sigma=\sigma_{0} \in i \mathbb{R}$, then, in the variable $\sigma-\sigma_{0}$ if $\sigma_{0} \neq 0$ or $u$ if $\sigma_{0}=0$, the pole is simple.

This is shown in Appendix [

Proposition 9. Condition (A) is satisfied for the potentials (1.8).

Proof. This is established in 96

\subsection{Asymptotic expansion of $\psi$ and Borel summability.}

Proposition 10. In the setting (1.7b) there exist $N \in \mathbb{N},\left\{\Gamma_{k}\right\}_{k \leq N}$, and $\left\{F_{\omega ; k}(t, x)\right\}_{k \leq N}$, $2 \pi / \omega$-periodic functions of $t$, such that, for $t>0$,

$$
\psi(t, x)=\sum_{j \in \mathbb{Z}} e^{i j \omega t} h_{j}(t, x)+\sum_{k=1}^{N} P_{k}(t) e^{-\Gamma_{k} t} F_{\omega ; k}(t, x)
$$

with $\Re \Gamma_{k} \geq 0$ for all $k \leq N, P_{k}(t)$ are polynomials in $t$, reducing to constants if $\Re \Gamma_{k}=0$, and the $h_{j}(t, x)$ have Borel summable power series in $t^{-1 / 2}$

$$
h_{j}(t, x)=\mathcal{L B} \sum_{k \geq k_{0}} h_{k j}(x) t^{-k / 2}
$$

with $k_{0} \geq 1$. 
Remark 11 (Borel summation). If $\tilde{f}$ is a formal power series, say in inverse powers of $t$, then $\tilde{F}=\mathcal{B} \tilde{f}$ is also a formal power series, defined as the term-wise inverse Laplace transform in $t$ of $\tilde{f}$. If (1) $\tilde{F}$ is convergent (2) its sum $F$ can be analytically continued along $\mathbb{R}^{+}$and (3) $\exists \nu$ s.t. $F(p) \in L^{1}\left(\mathbb{R}^{+}, e^{-\nu p} d p\right)$, then the Laplace transform $\mathcal{L} F$ is by definition the Borel sum of $\tilde{f}$ denoted by $\mathcal{L} \mathcal{B} \tilde{f}$. In our context we have, more precisely,

$$
h_{j}(t, x)=\int_{0}^{\infty} F_{j}(\sqrt{p}, x) e^{-p t} d p \sim \sum_{k} h_{k j}(x) t^{-k / 2}, t \rightarrow+\infty
$$

where the functions $F_{j}(s, x)$ are analytic in $s$ in a neighborhood of $\mathbb{R}^{+}$and for any $b \in \mathbb{R}$ there exist a constant $C$ such that for all $j$ and $p \in \mathbb{R}^{+}$,

$$
\sup _{p \geq 0 ;|x|<b}\left|F_{j}(\sqrt{p}, x) e^{-C|p|}\right| \leq f_{j}
$$

where the $f_{j}$ decay in $j$ faster than $j^{-2}$ under the assumption (1.4) and factorially if $\Omega$ is a trigonometric polynomial. Thus the function series in (3.23) converges (rapidly in the latter case).

The role of condition (A) is described in the following result.

Theorem 12. (i) If (A) holds, then on the right side of 3.24) and (3.23) we have

$$
k_{0} \geq 3 \text { and } \Re \Gamma_{k}>0 \text { for all } k \text {. }
$$

In particular we have complete ionization of the system. (See also Proposition 9 , as well as Proposition 14 and Remark 15 below.)

(ii) If (A) is not satisfied, then some $\Re \Gamma_{k}$ may vanish; the part of $\psi$ corresponding to these $\Gamma_{k}$ remains a spatially localized quasiperiodic function of $t$. Exceptionally, $k_{0}=1$ if (A) does not hold (see also [16] and Proposition [18).

The proofs of Proposition [10 and Theorem [12 are sketched in Appendix [D In one dimension a similar result is stated in [6].

\section{IONIZATION CONDITION FOR COMPACTLY SUPPORTED POTENTIALS}

For the setting (1.7a) we derive a technically convenient condition implying (A).

Assume $0 \neq v \in \mathcal{H}$ and $v=\mathfrak{C} v$. Then there exists a nontrivial solution in $\mathcal{H}$ to the system

$$
(-\Delta+\sigma+n \omega) y_{n}=-V y_{n}-\sum_{j \in \mathbb{Z}} \Omega_{j}(x) y_{n-j}
$$

We multiply (4.1) by $\bar{y}_{n}$, integrate over a ball $B$ containing $D$, sum over $n$ (which is legitimate since $y \in \mathcal{H}$ ) and take the imaginary part of the resulting expression. Noting that

$$
\begin{aligned}
\overline{\sum_{j, n \in \mathbb{Z}} \Omega_{j}(x) y_{n-j} \overline{y_{n}}}=\sum_{j, n \in \mathbb{Z}} \Omega_{-j} \bar{y}_{n-j} y_{n}=\sum_{j, n \in \mathbb{Z}} \Omega_{j} \bar{y}_{n+j} y_{n} & \\
& =\sum_{j, m \in \mathbb{Z}} \Omega_{j}(x) \overline{y_{m}} y_{m-j}
\end{aligned}
$$


so the sum (4.2) is real, we get

$$
\begin{aligned}
0=\Im\left(-\sigma \sum_{n \in \mathbb{Z}}\left\|y_{n}\right\|^{2}+\int_{B} \sum_{n \in \mathbb{Z}} d x \bar{y}_{n} \Delta y_{n}\right) \\
=-\Im \sigma \sum_{n \in \mathbb{Z}}\left\|y_{n}\right\|^{2}+\frac{1}{2 i} \int_{\partial B}\left(\sum_{n \in \mathbb{Z}} \bar{y}_{n} \nabla y_{n}-y_{n} \nabla \bar{y}_{n}\right) \cdot \mathbf{n} d S
\end{aligned}
$$

We take $d=3$ (the analysis is simpler in one or two dimensions). It is convenient to decompose $y_{n}$ using spherical harmonics; we write

$$
y_{n}=\sum_{l \geq 0,|m| \leq l} R_{n, l, m}(r) Y_{l}^{m}(\theta, \phi) .
$$

The last integral in (4.3), including the prefactor, then equals

$$
\begin{aligned}
-8 \pi i r_{B}^{2} \sum_{n \in \mathbb{Z}} \sum_{m, l}\left[\bar{R}_{n, m, l} R_{n, m, l}^{\prime}-\bar{R}_{n, m, l} R_{n, m, l}\right] \\
=-8 \pi i r_{B}^{2} \sum_{n \in \mathbb{Z}} \sum_{m, l} W\left[\bar{R}_{n, m, l}, R_{n, m, l}\right]
\end{aligned}
$$

where $r_{B}$ is the radius of $B$ and $W[f, g]$ is the Wronskian of $f$ and $g$. On the other hand, since $V$ and $\Omega$ are compactly supported, we have outside of $B$

$$
\Delta y_{n}-(\sigma+n \omega) y_{n}=0
$$

and then by (4.4), $R_{n, l, m}$ satisfy for $r>r_{B}$ the equation

$$
R^{\prime \prime}+\frac{2}{r} R^{\prime}-\frac{l(l+1)}{r^{2}} R=(\sigma+n \omega) R
$$

where we have suppressed the subscripts. Let $g_{n, l, m}=r R_{n, l, m}$. Then for the $g_{n, l, m}$ we get

$$
g^{\prime \prime}-\left[\frac{l(l+1)}{r^{2}}+(\sigma+n \omega)\right] g=0
$$

thus

and

$$
\bar{R} R^{\prime}=\frac{\bar{g} g^{\prime}}{r^{2}}-\frac{|g|^{2}}{r^{3}}
$$

$$
r^{2} W[\bar{R}, R]=W[\bar{g}, g]=: W_{n} .
$$

Multiplying (4.8) by $\bar{g}$, the conjugate of (4.8) by $g$ and subtracting, we get for $r>r_{B}$

$$
W_{n}^{\prime}=(\sigma-\bar{\sigma})|g|^{2}=2 i|g|^{2} \Im \sigma
$$

Remark 13. Simple estimates using equation (3.21), the definition (3.2) and 3.3) imply that, for some $c_{n}$,

$$
y_{n}(x)=\frac{e^{-\kappa_{n}|x|}}{|x|}\left(c_{n}(\theta, \phi)+O\left(|x|^{-1}\right)\right) \text { as }|x| \rightarrow \infty
$$

Let us consider two cases of (4.1).

Case (i): $\Im \sigma<0$. By Remark [13] we have

$$
g \sim C e^{-\kappa_{n} r}(1+o(1)) \text { as } r \rightarrow \infty
$$


There is a one-parameter family of solutions of (4.8) satisfying (4.13) and the asymptotic expansion can be differentiated [29. We assume, to get a contradiction, that there exist $n$ for which $g_{n} \neq 0$. For these $n$ we have, using (4.13), differentiability of this asymptotic expansion and (3.3) that

$$
\frac{1}{2 i} \lim _{r \rightarrow \infty}\left|g_{n}\right|^{-2} W_{n}=-\Im \kappa_{n}>0 \text {. }
$$

It follows from (4.11) and (4.14) that $\frac{1}{2 i} W_{n}$ is strictly positive for all $r>r_{B}$ and all $n$ for which $g_{n} \neq 0$. This implies that the last term in (4.3) is a sum of positive terms which shows that (4.3) cannot be satisfied.

Case (ii): $\Im \sigma=0$. For $n>0$ there exists only one solution $g$ of (4.8) which decays at infinity (cf. Remark [13 and the discussion in Case (i)), and since (4.8) has real coefficients this $g$ must be a (constant multiple of a) real function as well; therefore we have $W_{n}=0$ for $n \geq 0$.

For $n<0$, we use Remark 13 (and differentiability of the asymptotic expansion as in Case (i)) to calculate the Wronskian $W_{n}$ of $g, \bar{g}$ in the limit $r \rightarrow \infty$ : $W_{n}=$ $\left|c_{n}\right|^{2}(1+o(1))$. Since for $\Im \sigma=0, W_{n}$ is constant, cf. 4.11), it follows that $W_{n}$ is exactly equal to $\left|c_{n}\right|^{2}$. Thus, using (4.3) and (4.5) we have

$$
y_{n}(x)=0 \text { for all } n<0 \text { and }|x|>r_{B}
$$

Proposition 14. In the setting 1.7a), if (A) fails, then we have

$$
y_{n}(x)=0 \text { for all } n<0 \text { and } x \notin D
$$

Outside $D$ we have $\mathfrak{O} y_{n}=0$, where $\mathfrak{O}$ is the elliptic operator $-\Delta+\sigma+n \omega$. The proof follows immediately from (4.15), by standard unique continuation results [17, [22, 28] ( in fact, $\mathfrak{O}$ is analytic hypo-elliptic).

Remark 15. Proposition 14 points toward generic ionization under time periodic forcing. Indeed, we see from (4.16) that equations (4.1) are formally overdetermined when $n<0$ ( $y_{n}$ is in the domain of $\Delta$ so that, in 4.1), the function and "one derivative" are given on the boundary) and are expected, generically, not to have nontrivial solutions even if $y_{n}$ had to satisfy (3.12) for $n<0$ only.

The latter reduced problem is relatively easier to study and we used it to show that (A) holds in a number of settings, including the potential (1.8), see [6].

Remark 16. There do in fact exist nongeneric potentials (though not in the class [1.3)) for which ionization fails [8, 21, 24].

\section{Connection With Floquet theory, Continued}

Proposition 17. If $u$ is an eigenfunction or resonance ${ }^{4}$ of the operator $K$ defined in 2.7) such that $u \in L^{2}\left(\mathbb{R}^{3} \times S_{2 \pi / \omega}^{1}\right)$, then $u=\mathfrak{C} u$ in $\mathcal{H}$ and so (A) fails.

The proof is an immediate consequence of Proposition 28] in Appendix E Conversely, we have the following result.

Proposition 18. We assume the setting (1.7a).

(i) If $v=\mathfrak{C} v$ for some $\sigma_{0} \in(0, \omega)$ and $v \in \mathcal{H}$, then $v \in l^{2}\left(L^{2}\left(\mathbb{R}^{d}\right)\right)$ thus it is an eigenfunction of $K$.

(ii) If $v=\mathfrak{C} v$ for $\sigma_{0}=0$ and $v \in \mathcal{H}$, then, for $d=3$, $v$ is of the form $v=$ $C|x|^{-1} \delta_{n 0}+\tilde{v}(x)$ where $\tilde{v} \in l^{2}\left(L^{2}\left(\mathbb{R}^{3}\right)\right.$ ) (resonance of $K$ ).

\footnotetext{
${ }^{4}$ See 16 and Proposition 18 (ii).
} 
Proof of Proposition [18, For the same reasons as before, we focus on $d=3$.

(i) We see from (3.6), (3.4) and (4.16) that for all $n$ we have $y_{n} \in L^{2}\left(\mathbb{R}^{3}\right)$. Furthermore, a straightforward calculation shows that $\left\|y_{n}\right\|_{L^{2}\left(\mathbb{R}^{3}\right)} \leq C\left\|y_{n}\right\|_{D}$ where $C$ is independent of $n$. Proposition 28] in Appendix E gives the necessary estimates in $n$ to complete the proof in this case.

(ii) For $n \neq 0$ we have, for the same reasons as in (i), $y_{n} \in L^{2}\left(\mathbb{R}^{3}\right)$. But now, at $n=0$, since $\sigma_{0}=0$ the Green function (3.4) does not have enough decay to ensure $y_{0} \in L^{2}\left(\mathbb{R}^{3}\right)$. We have instead, for $x^{\prime} \in D$ and $|x| \rightarrow \infty, G_{0}\left(x-x^{\prime}\right)=$ $\frac{1}{4 \pi}|x|^{-1}+O\left(|x|^{-2}\right)$. The statement now follows from (3.6) and (3.11).

\section{Example (1.8)}

To show that it can be effectively checked whether (4.16) can be nontrivially satisfied, we consider the example (1.8). It is convenient to Fourier transform the system (4.1) in $x$. In view of (4.16), for $n<0, y_{n}=0$ outside $D$. We then have, for $n<0$,

and

$$
\check{y}_{n}:=\int_{\mathbb{R}^{3}} y_{n} e^{-i k \cdot x} d x=\int_{D} y_{n} e^{-i k \cdot x} d x
$$

$$
-k^{2} \check{y}_{n}=-k^{2} \int_{\mathbb{R}^{3}} y_{n} e^{-i k \cdot x} d x=\int_{\mathbb{R}^{3}} \Delta y_{n} e^{-i k \cdot x} d x=\int_{D} \Delta y_{n} e^{-i k \cdot x} d x
$$

For the setting (1.8) and $n<-1$, (4.1) reads

$$
\left(k^{2}+\sigma+n \omega\right) \check{y}_{n}=-V_{D} \check{y}_{n}+i \Omega_{D}\left(\check{y}_{n+1}-\check{y}_{n-1}\right)
$$

Remark 19. For $n \leq-1$, the functions $\check{y}_{n}$ are entire of exponential order one; more precisely, if $B$ is a ball containing $D$ we have

$$
\left|\check{y}_{n}(k)\right| \leq \sqrt{\operatorname{Vol}(D)} e^{|k| r_{B}}\left\|y_{n}\right\|_{L^{2}(D)}
$$

Proof. This follows immediately from the definition of $\check{y}$. (See also 32 for a comprehensive characterization of the Fourier transform of a compactly supported distribution.)

Proposition 20. The generating function

$$
Y(k, z)=\sum_{m \geq 0} \check{y}_{-m-2}(k) z^{m}
$$

is entire in $k$ and analytic in $z$ for $|z|<1$.

Proof. Since $y \in \mathcal{H}$ we have

$$
\left\|y_{n}\right\|_{L^{2}(D)} \leq \text { const }|n|^{-3 / 2}
$$

Using Remark 19 the conclusion follows.

A straightforward calculation shows that $Y$ satisfies the equation

$$
M Y-z \frac{\partial Y}{\partial z}-i \beta\left(z-\frac{1}{z}\right) Y=i \beta \check{y}_{-1}+i \beta \frac{\check{y}_{-2}}{z}
$$

where

$$
M=\omega^{-1}\left(k^{2}+\sigma-2 \omega+V_{D}\right)
$$

and $\beta=\Omega_{D} / \omega$. The solution of (6.7) is

$$
Y=z^{M} e^{-i \beta\left(z+z^{-1}\right)}\left[C(k)-i \beta \int_{0}^{z} e^{i \beta\left(s+s^{-1}\right)}\left(\frac{\check{y}_{-1}}{s^{M+1}}+\frac{\check{y}_{-2}}{s^{M+2}}\right) d s\right]
$$


where the integral follows a path in which 0 is approached along the negative imaginary line.

Remark 21. Proposition 20 implies $C(k) \equiv 0$.

Proof. It is easy to check that otherwise the limit of $Y(k, z)$ as $z \rightarrow 0$ along $i \mathbb{R}^{-}$ would not exist.

Thus

$$
Y(k, z)=-i \beta z^{M} e^{-i \beta\left(z+z^{-1}\right)} \int_{0}^{z} e^{i \beta\left(s+s^{-1}\right)}\left(\frac{\check{y}_{-1}(k)}{s^{M+1}}+\frac{\check{y}_{-2}(k)}{s^{M+2}}\right) d s
$$

We now use the nontrivial monodromy of $Y$ on the Riemann surface of $\log z$, following from the integral representation (6.10). Analytic continuation around the origin gives

$$
i \beta^{-1} e^{i \beta\left(z+z^{-1}\right)}\left(Y\left(\cdot, z e^{2 \pi i}\right)-Y(\cdot, z)\right)=\check{y}_{-1} \oint_{\mathcal{C}} \frac{e^{i \beta\left(s+s^{-1}\right)}}{s^{M+1}} d s+\check{y}_{-2} \oint_{\mathcal{C}} \frac{e^{i \beta\left(s+s^{-1}\right)}}{s^{M+2}} d s
$$

where $\mathcal{C}$ is the curve shown in Fig. 1. Let

$$
F(M)=\oint_{\mathcal{C}} \frac{e^{i \beta\left(s+s^{-1}\right)}}{s^{M}} d s
$$

Proposition 22. We have

$$
\check{y}_{-1}(k) F(M+1)+\check{y}_{-2}(k) F(M+2)=0
$$

Proof. This follows immediately from the discussion above.

Proposition 23. For every large $N \in \mathbb{N}, F(z)$ has exactly one zero of the form $z_{N}=N+o\left(N^{0}\right)$. For large $N$ we have $F\left(1+z_{N}\right) \neq 0$.

Proof. It turns out that $F(M)$ is a Bessel function of order $M$ evaluated at 2 and a proof can be given based on this representation. However, in view of later generalizations we prefer to give a more general argument that does not rely on explicit representations.

Let $M=N+\zeta$ with $N$ a large positive integer, $\zeta$ complex with $|\zeta|=\epsilon$ and $\epsilon$ positive and small. Let $C_{1}$ be the counterclockwise circle $\{z:|z|=1\}$ and $L$ the segment $[0,-i]$; we write

$$
\begin{aligned}
F(N+\zeta)=(1 & \left.-e^{-2 \pi i \zeta}\right) \int_{L} \frac{e^{i \beta\left(s+s^{-1}\right)}}{s^{M}} d s+\int_{C_{1}} \frac{e^{i \beta\left(s+s^{-1}\right)}}{s^{M}} d s \\
& =2 \pi i \zeta\left(1+O\left(\zeta^{-1}\right)\right) \int_{L} \frac{e^{i \beta\left(s+s^{-1}\right)}}{s^{M}} d s+\int_{C_{1}} \frac{e^{i \beta\left(s+s^{-1}\right)}}{s^{M}} d s
\end{aligned}
$$

where in the integral along $L$ the principal branch of the log is used. The integral over $L$ can be estimated with Watson's Lemma, see e.g. 3]

$$
2 \pi i \zeta \int_{L} \frac{e^{i \beta\left(s+s^{-1}\right)}}{s^{M}} d s=i \zeta(2 \pi)^{3 / 2} \beta^{1-N} N^{N-3 / 2} e^{-N} e^{-\zeta \ln (\beta / N)}\left(1+o\left(N^{0}\right)\right)
$$

By the Riemann-Lebesgue lemma, $\int_{C_{1}} \rightarrow 0$ as $N \rightarrow \infty$. We get

$$
F(N+\zeta)=i^{M} \zeta(2 \pi)^{3 / 2} \beta^{1-N} N^{N-3 / 2} e^{-N} e^{-\zeta \ln (\beta / N)}\left(1+o\left(N^{0}, \zeta^{0}\right)\right)+o\left(N^{0}\right)
$$




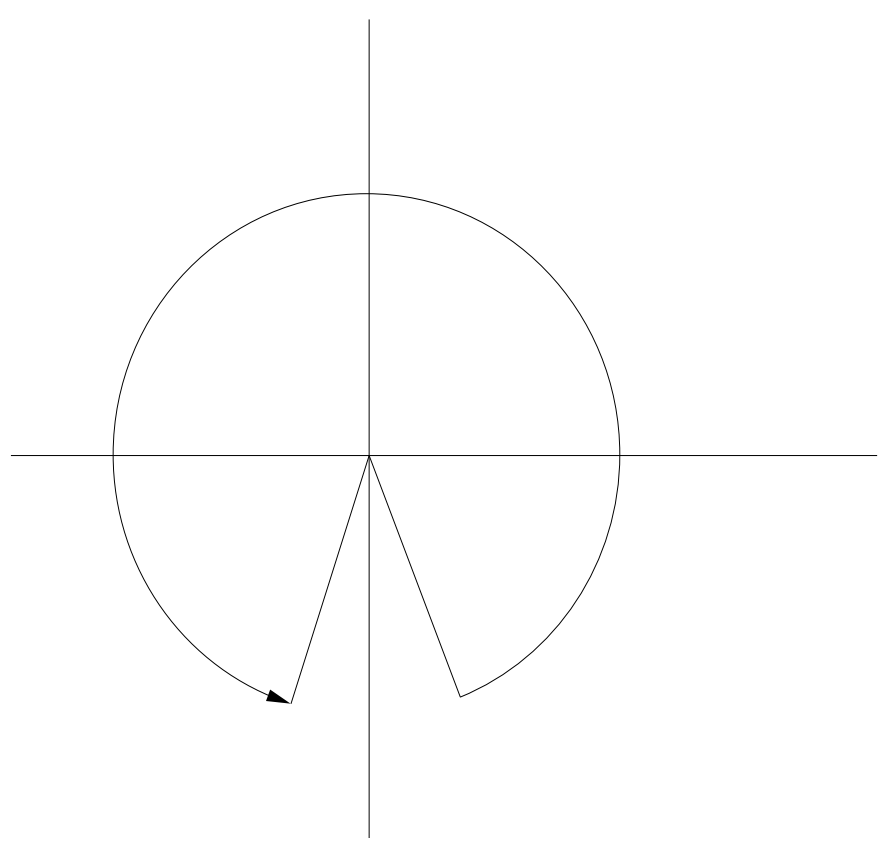

Figure 1. The curve $\mathcal{C}$.

The existence of a unique simple zero at some $N+\zeta_{N}$ with $\left|\zeta_{N}\right|<\epsilon$ is a consequence of the argument principle.

The position of $\zeta_{N}$ can be found more accurately as follows. We have

$$
\zeta_{N}=\frac{\zeta_{N}}{1-e^{-2 \pi i \zeta_{N}}} \int_{C_{1}} \frac{e^{i \beta\left(s+s^{-1}\right)}}{s^{N+\zeta_{N}}} d s\left(\int_{L} \frac{e^{i \beta\left(s+s^{-1}\right)}}{s^{N+\zeta_{N}}} d s\right)^{-1}
$$

from which it follows that $\zeta_{N}=o\left(\right.$ const $^{N} / N$ !) which readily implies that (6.16) is contractive and that

$$
\zeta_{N}=\frac{1}{2 \pi i} \int_{C_{1}} \frac{e^{i \beta\left(s+s^{-1}\right)}}{s^{N}} d s\left(\int_{L} \frac{e^{i \beta\left(s+s^{-1}\right)}}{s^{N}} d s\right)^{-1}\left(1+o\left(N^{0}\right)\right)
$$

Using (6.15) and the fact that the first integral in (6.17) gives the Laurent coefficients of $e^{i \beta\left(s+s^{-1}\right)}$ which can be independently estimated from the series expansion, we find that, with constants that can be calculated,

$$
\left|\zeta_{N}\right|=c_{1} c_{2}^{N} N^{-2 N+c_{3}}\left(1+o\left(N^{0}\right)\right)
$$

Thus $\zeta_{N+1} / \zeta_{N} \rightarrow 0$ as $N \rightarrow \infty$ and the second part of the Proposition follows. 
Proposition 24. Relation (6.13), with $\check{y}_{-1}(k), \check{y}_{-2}(k)$ entire of exponential order one (cf. Remark 19) implies

$$
\check{y}_{-1}(k)=\check{y}_{-2}(k)=0 \forall k \in \mathbb{C}^{3}
$$

and then

$$
y_{n}(x)=0 \forall n \in \mathbb{Z} \text { and almost all } x \in \mathbb{R}^{d}
$$

Proof. Propositions 22 and 23 and (6.8) imply that $\check{y}_{-1}(k)$ has at least const $R^{2}$ zeros in a disk of large radius $R$. Since $\check{y}_{-1}(k)$ is an entire function of exponential order one, it follows that $\check{y}_{-1} \equiv 0$ (see, e.g. 25). By (6.13) we have $\check{y}_{-2} \equiv 0$, so that $y_{-1} \equiv y_{-2} \equiv 0$.

In the present model (4.1) reads

$$
(-\Delta+\sigma+n \omega) y_{n}=-V_{D} \chi_{D} y_{n}-i \Omega_{D} \chi_{D}\left(y_{n+1}-y_{n-1}\right)
$$

and 6.21) with $n=-1$ and $n=-2$ implies $\chi_{D} y_{0}=0, \chi_{D} y_{-3}=0$ respectively; inductively $\chi_{D} y_{n}=0$ for all $n$. Then $(-\Delta+\sigma+n \omega) y_{n}=0$ in $\mathbb{R}^{d} \backslash \partial D$. Since, by Proposition 18 $y_{n} \in L^{2}\left(\mathbb{R}^{3}\right)$ we see (for instance by taking the Fourier transform) that $\left\|y_{n}\right\|=0$.

Remark 25. It can be shown that condition (A) holds with $V_{D} \chi_{D}$ replaced by $V_{D} \chi_{D}+V_{1}(x)$ where $V_{1}(x)$ is bounded, not necessarily constant, with compact support disjoint from $D$. On the support of $V_{1}, \Omega$ is zero and it can be seen that for $n$ sufficiently negative $y_{n}$ is zero on the support of $V_{1}$. From this point on the arguments are very similar, but we will not pursue this here.

\section{Compactness}

7.1. Compactness of the operator $\mathfrak{g}_{n}$ defined in (3.6). The case $d=1$ is discussed in Appendix A For $d=2,3$ compactness follows from Theorem VI.23, Vol. 1, pp. 210 of $\left[23\right.$ ( for $d=3$, note that $e^{-\kappa_{n}|x-y|} /|x-y| \in L^{2}(D \times D)$ ).

7.2. Compactness of $\mathfrak{C}$. The property (3.14) is mentioned in Appendix A of 1 . We include here an elementary proof of (7.1) below (which also can be refined without serious difficulty to yield the sharper estimate (3.14)).

Lemma 26. We have

$$
\left\|\mathfrak{g}_{n}\right\| \longrightarrow 0 \quad \text { as }|n| \rightarrow \infty
$$

(where $\|\cdot\|$ is the $L^{2}(D) \mapsto L^{2}(D)$ operator norm) uniformly in $u$ in the region $S_{\omega, \epsilon, A}=\left\{u:|u|<A,\left|\Re\left(u^{2}\right)\right|<\omega-\epsilon\right\}$, where $A>0$ and $\epsilon$ is any small positive number.

Proof. Relation (7.1) follows from a general result by Agmon 1] which provides estimates on the rate of convergence. We give below an elementary proof in our case.

We prove the result for $d=3$ (the proof is simpler in $d=1,2$, noting that for large $x$ with $\arg x \in(-\pi, \pi)$ we have $\left.K_{0}(x)=\sqrt{\frac{\pi}{2}} e^{-x} x^{-1 / 2}(1+o(1))\right)$. Define

$$
Q_{n}\left(x^{\prime}, x^{\prime \prime}\right)=\int_{D} d x \frac{e^{-\kappa_{n}\left|x^{\prime}-x\right|-\bar{\kappa}_{n}\left|x^{\prime \prime}-x\right|}}{\left|x^{\prime}-x\right|\left|x^{\prime \prime}-x\right|}
$$


We have

$$
\begin{aligned}
\left\|\mathfrak{g}_{n}\right\|^{2}=\sup _{\|f\|=1} \int_{D^{2}} Q_{n}\left(x^{\prime}, x^{\prime \prime}\right) f\left(x^{\prime}\right) \overline{f\left(x^{\prime \prime}\right)} & d x^{\prime} d x^{\prime \prime} \\
& \leq\left(\int_{D^{2}}\left|Q_{n}\left(x^{\prime}, x^{\prime \prime}\right)\right|^{2} d x^{\prime} d x^{\prime}\right)^{1 / 2}
\end{aligned}
$$

The last integral goes to zero as $|n| \rightarrow \infty$. To see that, note that

$$
\sqrt{n \omega+u^{2}}=\sqrt{n \omega}+O\left(n^{-1 / 2}\right) ; \text { as } n \rightarrow+\infty
$$

and using the triangle inequality we get

$$
\begin{aligned}
&\left|Q_{n}\left(x^{\prime}, x^{\prime \prime}\right)\right| \leq \text { Const } e^{-\sqrt{n \omega}\left|x^{\prime}-x^{\prime \prime}\right|} \int_{D} \frac{d x}{\left|x^{\prime}-x\right|\left|x^{\prime \prime}-x\right|} \\
& \leq \text { Const } e^{-\sqrt{n \omega}\left|x^{\prime}-x^{\prime \prime}\right|}
\end{aligned}
$$

and the conclusion, for $n \rightarrow+\infty$ follows by dominated convergence. We now focus on large negative $n$. Since

$$
\sqrt{n \omega+u^{2}}=-i \sqrt{|n| \omega}+O\left(n^{-1 / 2}\right) ; \quad \text { as } n \rightarrow-\infty
$$

it is easy to check that (7.1) follows once we show that $\left\|\mathfrak{g}_{[\nu]}\right\| \rightarrow 0$ as $\nu \rightarrow \infty$ where $\left\|\mathfrak{g}_{[\nu]}\right\|$ is obtained by replacing $\kappa_{n}$ with $i \nu$ in the definition of $\mathfrak{g}_{n}$. We first show, with an analogous definition of $Q_{[\nu]}$, that

$$
\sup _{x, x^{\prime} \in D, \nu \in \mathbb{R}}\left|Q_{[\nu]}\left(x, x^{\prime}\right)\right|=Q_{0}<\infty
$$

Indeed, we choose a ball $B_{b}$ centered at $x^{\prime}$ of radius $b$ large enough so that it contains $D$ and write the integrals (7.2) in spherical coordinates centered at $x^{\prime}$ with $x^{\prime \prime}$ on the $z$ axis; in these coordinates $\left|x-x^{\prime}\right|=r$ and $\left|x-x^{\prime \prime}\right| \geq d(x, O z)=r \sin \theta$ and thus

$$
\left|Q_{[\nu]}\left(x, x^{\prime}\right)\right| \leq \int_{B_{b}} \frac{d x}{\left|x-x^{\prime}\right|\left|x-x^{\prime \prime}\right|} \leq \int_{B_{b}} d r d \theta d \phi \leq 4 \pi b
$$

Let $\rho\left(x ; x^{\prime}, x^{\prime \prime}\right)=\left|x-x^{\prime}\right|-\left|x-x^{\prime \prime}\right|$. We then have $\left|\rho\left(x ; x^{\prime}, x^{\prime \prime}\right)\right| \leq\left|x^{\prime}-x^{\prime \prime}\right|$ and we get

$$
Q_{[\nu]}\left(x^{\prime}, x^{\prime \prime}\right)=\int_{D} d x \frac{e^{i \nu \rho\left(x ; x^{\prime}, x^{\prime \prime}\right)}}{\left|x^{\prime}-x\right|\left|x^{\prime \prime}-x\right|}=\int_{-\left|x^{\prime \prime}-x^{\prime}\right|}^{\left|x^{\prime \prime}-x^{\prime}\right|} e^{i \nu \rho} d \mu(\rho)
$$

where the positive measure $\mu$ is defined by

$$
\mu(A)=\mu_{x^{\prime}, x^{\prime \prime}}(A)=\int_{\{x: \rho(x) \in A\} \cap D} \frac{d x}{\left|x^{\prime}-x\right|\left|x^{\prime \prime}-x\right|}
$$

Since the integrand in (7.9) is in $L^{1}$, the measure $\mu$ is absolutely continuous with respect to the Lebesgue measure $m$. We let $h\left(\rho ; x^{\prime}, x^{\prime \prime}\right)=\frac{d \mu}{d m}$; then $h \in L^{1}$ and we get

$$
Q_{[\nu]}\left(x^{\prime}, x^{\prime \prime}\right)=\int_{-\left|x^{\prime \prime}-x^{\prime}\right|}^{\left|x^{\prime \prime}-x^{\prime}\right|} e^{i \nu \rho} h\left(\rho ; x^{\prime}, x^{\prime \prime}\right) d \rho
$$


By the Riemann-Lebesgue lemma we have ${ }^{5}$

$$
Q_{[\nu]}\left(x^{\prime}, x^{\prime \prime}\right) \rightarrow 0 \text { as } \nu \rightarrow \infty
$$

Now (7.7), (7.11) and again dominated convergence implies $\left\|\mathfrak{g}_{[\nu]}\right\| \rightarrow 0$ as $\nu \rightarrow \infty$ completing the proof.

Lemma 27. Under the assumption 1.7a), the operator $\mathfrak{C}$ is compact on $\mathcal{H}$ and analytic in $u$ in $S_{\omega, \epsilon, A}$, cf. Lemma [26]

Indeed, $\mathfrak{C}$ is the norm limit (3.19), uniform in $u \in S_{\omega, \epsilon, A}$, where $\mathfrak{C}_{N}$ are compact by Lemma 26] and analytic as explained in the proof of Lemma $[5$ We note that the operator

$$
\left\|\sum_{j \in \mathbb{Z}} \Omega_{j} S^{-j}\right\|
$$

is bounded in $\mathcal{H}$. Indeed, if we write $\langle n\rangle:=1+|n|$ we have, for $(n, j) \in \mathbb{Z}^{2}$ $\langle n\rangle \leq\langle j\rangle\langle n-j\rangle$ and

$$
\begin{aligned}
\sum_{n \in \mathbb{Z}}\langle n\rangle^{\gamma}\left|\sum_{j \in \mathbb{Z}} \Omega_{j} y_{n-j}\right|^{2} \leq \sum_{n \in \mathbb{Z}}\left(\sum_{j \in \mathbb{Z}}\langle j\rangle^{\gamma / 2}\left|\Omega_{j}\right|\langle n-j\rangle^{\gamma / 2}\left|y_{n-j}\right|\right)^{2} & \\
= & \sum_{j_{1}, j_{2} \in \mathbb{Z}}\left\langle j_{1}\right\rangle^{\frac{\gamma}{2}}\left|\Omega_{j_{1}}\right|\left\langle j_{2}\right\rangle^{\frac{\gamma}{2}}\left|\Omega_{j_{2}}\right| \sum_{n \in \mathbb{Z}}\left\langle n-j_{1}\right\rangle^{\frac{\gamma}{2}}\left|y_{n-j_{1}}\right|\left\langle n-j_{2}\right\rangle^{\frac{\gamma}{2}}\left|y_{n-j_{2}}\right| \\
& \leq\|y\|_{l_{\gamma}^{2}}^{2}\left\|\langle j\rangle^{\gamma / 2} \Omega_{j}\right\|_{1}^{2} \leq C\|y\|_{l_{\gamma}^{2}}^{2}
\end{aligned}
$$

by (1.4).

\section{Appendixes}

A. Compact operator formulation and proof of Lemma 2 for $d=1$. We can assume without loss of generality $D \subset[-1,1]$. For $n=0$ we choose some large positive $a$ such that $\sin 2 \sqrt{a} \neq 0$, denote by $f_{ \pm}(x)$ the functions $e^{\mp u x}$ and let $\psi_{+}$ be the solution of the equation

$$
-\psi^{\prime \prime}+\left(a \chi_{[-1,1]}+u^{2}\right) \psi=0
$$

(see Remark (4) with initial condition $\psi_{+}(1)=f_{+}(1), \psi_{+}^{\prime}(1)=f_{+}^{\prime}(1)$, and similarly let $\psi_{-}$be the solution of (8.1) with initial condition $\psi_{-}(-1)=f_{-}(-1), \psi_{-}^{\prime}(-1)=$ $f_{-}^{\prime}(-1)$. Since both the equation and the initial conditions are analytic in $u$ at $u=0$, so are the solutions $\psi_{ \pm}$and their Wronskian $W(u)$. It can be checked that $W(0)=\sqrt{a} \sin 2 \sqrt{a} \neq 0$. In fact, taking $\tau_{a}=\sqrt{a-u^{2}}$ we have

$$
\left.\psi_{ \pm}(x)=\tau_{a}^{-1} e^{-u}\left[\tau_{a} \cos \left(\tau_{a} x \mp \tau_{a}\right) \mp u \sin \left(\tau_{a} x \mp \tau_{a}\right)\right)\right]
$$

In a neighborhood of $u=0$ we write for $n \neq 0$ the same integral expression (3.11), while for $n=0$ we write

$$
y_{0}=\mathfrak{g}_{0, a} \psi_{1,0}-\mathfrak{g}_{0, a}\left(V+a \chi_{[-1,1]}\right) y_{0}+i \mathfrak{g}_{0, a}\left(\sum_{j \in \mathbb{Z}} \Omega_{j} S^{-j} y\right)_{n}
$$

\footnotetext{
${ }^{5}$ Noting that the estimate of the norm of $\mathfrak{g}_{n}$ can only increase if extended to $L^{2}(B)$ where $B$ is a ball containing $D$, and that $h$ calculated in $B$ is piecewise smooth the max of $Q_{[\nu]}$ can be in fact bounded by an inverse power of $\nu$; we do not however need this refinement here.
} 
where

$$
W(u)\left(\mathfrak{g}_{0, a} f\right)(x)=\psi_{+}(x) \int_{-1}^{x} \psi_{+}(s) f(s) d s-\psi_{-}(x) \int_{1}^{x} \psi_{-}(s) f(s) d s
$$

With the same conventions, we now write the integral system in the form (3.11). Compactness and analyticity are now shown in the same way as for $d=2,3$.

B. Proof of Lemma 3. Let $\sigma=\sigma_{0}-2 i \tau$ where $\sigma_{0} \in[0, \omega)$ and $\tau>0$. We show that

$$
\|\mathfrak{C}\| \rightarrow 0 \text { as } \tau \rightarrow \infty
$$

and uniqueness follows by contractivity. The calculation leading to (8.5) is quite straightforward, but we provide it for convenience. In $d=1,2$ the estimate follows from the behavior of the Green function for large argument. We then focus on $d=3$. By (3.3) we have

$$
\Re\left(\kappa_{n}\right)=\left(\frac{1}{2}\left(\left(\sigma_{0}+n \omega\right)^{2}+\sigma_{0}+n \omega\right)^{1 / 2}+\tau\right)^{1 / 2}
$$

For $n>0$ we then have $\Re \kappa_{n}>\sqrt{n \omega}$ and the same calculation as for (7.5) shows that

$$
\left\|\mathfrak{g}_{n}\right\| \underset{L^{2}(B)}{\longrightarrow} 0 \text { as } n \rightarrow+\infty
$$

uniformly in $\tau$. For $n<0$ (8.6) gives

$$
\left|Q_{n}\left(x^{\prime}, x^{\prime \prime}\right)\right| \leq\left|Q_{\nu}\left(x^{\prime}, x^{\prime \prime}\right)\right|
$$

where

$$
-\nu:=\Im\left(\kappa_{n}\right) \rightarrow \infty \text { as } n \rightarrow-\infty
$$

and now (7.11) shows that

$$
\left\|\mathfrak{g}_{n}\right\| \underset{L^{2}(B)}{\longrightarrow} 0 \text { as } n \rightarrow-\infty
$$

uniformly in $\tau$. We choose then $n_{0}$ large enough so that

$$
\sup _{n \geq n_{0}, \tau>0}\left\|\mathfrak{g}_{n}\right\| \leq \epsilon
$$

For $\tau$ large enough we have, still from (8.6),

$$
\Re\left(\kappa_{n}\right)>\frac{1}{2} \tau^{1 / 2} ; \quad-n_{0} \leq n \leq n_{0}
$$

Choosing a ball $B$ centered at $x$ containing $D$, we then have for large $\tau$ and some constants independent of $f, \tau, x$ and $n \in\left(-n_{0}, n_{0}\right)$, with the notation $\alpha=\frac{1}{2} \tau^{1 / 2}$,

$$
\begin{aligned}
\left|\left(\mathfrak{g}_{n} f\right)(x)\right| \leq \| \frac{e^{-\alpha\left|x-x^{\prime}\right|}}{\left|x-x^{\prime}\right|} & \left\|_{L^{2}(D)}\right\| f \|_{L^{2}(D)} \\
& \leq C_{1}\|f\|_{L^{2}(D)}\left\|\frac{e^{-\alpha\left|x-x^{\prime}\right|}}{\left|x-x^{\prime}\right|}\right\|_{B} \leq \frac{C_{2}}{\tau}\|f\|_{L^{2}(D)} \leq \epsilon
\end{aligned}
$$

and the conclusion follows. 
C. Proof of Lemma 8, Assume $\sigma_{0}$ is a value of $\sigma$ where invertibility of $I-\mathfrak{C}\left(\sigma_{0}\right)$ fails. Then $\sigma_{0} \in[0, \omega)$. By the Fredholm alternative we know that $I-\mathfrak{C}(\sigma)$ is invertible in some punctured neighborhood of $\sigma_{0}$ where the solution of (3.11) is meromorphic.

(i): $\sigma_{0} \neq 0$. Denote $\zeta=\sigma-\sigma_{0}$. We rewrite 3.11) in a suitable way near $\sigma_{0}$. We have from (3.12)

$$
\left(-\Delta+\sigma_{0}+n \omega\right) y_{n}=-i \psi_{2, n}-V y_{n}-\zeta y_{n}+\sum_{j \in \mathbb{Z}} \Omega_{j}(x)\left(S^{-j} y\right)_{n}
$$

which we write symbolically

$$
\mathfrak{W} y=-\zeta y-i \psi_{2, n}
$$

and from (3.12) and (3.11) we have ${ }^{* * * * * * * * * * * * *}$

$$
y_{n}=-i \mathfrak{g}_{n} \psi_{2, n}-\mathfrak{g}_{n}\left[V y_{n}-\zeta y_{n}-\sum_{j \in \mathbb{Z}} \Omega_{j}\left(S^{-j} y\right)_{n}\right]
$$

implying the following version of (3.11), with evident notation,

$$
y=y_{0}+\zeta \mathfrak{g} y+\mathfrak{C}\left(\sigma_{0}\right) y
$$

On the other hand,

$$
y=\sum_{j=-M}^{\infty} c_{j} \zeta^{j}
$$

with the coefficients $c_{j} \in \mathcal{H}$. Assume, to get a contradiction, that $M \geq 2$. Inserting in (8.17) we get

$$
\begin{aligned}
c_{-M} & =\mathfrak{C}\left(\sigma_{0}\right) c_{-M} \\
c_{-M+1} & =\mathfrak{C}\left(\sigma_{0}\right) c_{-M+1}+\mathfrak{g} c_{-M} \\
\ldots &
\end{aligned}
$$

In differential form we have,

$$
\begin{aligned}
\mathfrak{W} c_{-M} & =0 \\
\mathfrak{W} c_{-M+1} & =-c_{-M}
\end{aligned}
$$

By (8.19) and Proposition 18 we have $c_{-M} \in l^{2}\left(L^{2}\left(\mathbb{R}^{d}\right)\right):=\mathcal{H}_{1}$ (in fact, $\left(c_{-M}\right)_{n}$ decay at least exponentially in $|x|)$. On the other hand we then have from [8.20] and noting the formal self-adjointness of $\mathfrak{W}$,

$$
\left\langle c_{-M}, c_{-M}\right\rangle=-\left\langle c_{-M}, \mathfrak{W} c_{-M+1}\right\rangle=-\left\langle\mathfrak{W} c_{-M}, c_{-M+1}\right\rangle=0
$$

which is a contradiction.

(ii) $\sigma_{0}=0$ : there are two differences w.r.t case (i): (a) meromorphicity and Laurent expansions now use the variable $u=\sqrt{\sigma}$; and (b) $c_{-M}$ is not necessarily in $\mathcal{H}_{1}$ so we work with $\mathcal{H}_{B}=l^{2}\left(L^{2}(B)\right)$ for large enough $B$. These differences can be dealt with straightforwardly, so we just outline the main steps. The Laplacian is the only ingredient of $\mathfrak{W}$ not formally self-adjoint in $\mathcal{H}_{B}$. Integration by parts, implicit in (8.22) brings in boundary terms of the form

$$
\int_{\partial B} f \nabla g \cdot d S
$$

where $f$ and $g$ are $c_{-M}$ or $c_{-M+1}$. Both $f$ and $g$ have decay $|x|^{-1}$ and this behavior is differentiable, as is manifest from (8.19), (3.4), and (3.6). The contribution 
from the integrals (8.23) is thus $O\left(r_{B}^{-1}\right)$ which equals the norm $\left\|c_{-M}\right\|_{\mathcal{H}_{B}}$, clearly nondecreasing in $r_{B}$. This again forces $c_{-M}=0$, a contradiction.

D. Sketch of the proof of Proposition 10 and Theorem 12, We first show Theorem 12 (i). The contour of the inverse Laplace transform can be deformed as shown in Fig. 2. Pushing the contour of integration to the left brings in residues due to the meromorphic integrand, and since the kernel of the inverse Laplace transform is $e^{p t}$, residues in the left half plane give rise to decaying exponentials in $\psi(x, t)$. Uniform bounds on the Green function as $p \rightarrow-\infty$ are easy to prove. Consequently, there are only finitely many arrays of poles of $\hat{\psi}$. The contour of integration in the inverse Laplace transform can be pushed all the way to $-\infty$ in view of the exponential decay of the kernel $e^{p t}$. We are left with integrals along the sides of the cuts which, after the change of variable $p \leftrightarrow-p$ (or $p \leftrightarrow-p e^{i \alpha}$ if poles exist on the cuts), are seen to be Laplace transforms. Since $\hat{\psi}$ is analytic in $\sqrt{p+i n \omega}$, the contour deformation result shows, ipso facto, Borel summability of the asymptotic series of $\psi(x, t)$ for large $t$.

The general case is proved in a very similar way, using Lemma 8 If (A) does not hold, then some of the poles of the meromorphic function $y_{n}(\sigma)$ can be on the segment $(-\omega, \omega)$. If a pole is placed at $\sigma=0$, then analyticity in $u$ in the operator entails a singularity of the form $\sigma^{-1 / 2} A(\sigma)+B(\sigma)$ with $A$ and $B$ analytic, whence the conclusion.

\section{E. Estimates needed for Proposition 17] and Proposition 18,}

Proposition 28. Let $y$ be a solution in $l^{2}\left(L^{2}(B)\right)$ of the homogeneous equation associated to (3.12). Under the assumptions (1.7b), we have

$$
\left\|y_{j}\right\|_{L^{2}(B)}=O\left(j^{-2}\right) \text { as }|j| \rightarrow \infty
$$

Let $\epsilon$ be small enough and choose $j_{0}>0$ large enough (the proof is similar for $j_{0}<0$ ) so that $\left\|\mathfrak{g}_{j}\right\|_{L^{2}(B)}<\epsilon$ for all $j \geq j_{0}$, see (7.1). We consider the Banach space $\mathcal{B}_{j_{0}}$ of sequences of functions $\left\{y_{j}\right\}_{j \geq j_{0}}$ defined on $B$ for which the norm

$$
\|y\|_{j_{0}}:=\sup _{j \geq j_{0}} j^{2}\left\|y_{j}\right\|_{L^{2}(B)}
$$

is finite. For $j>j_{0}$ we write the homogeneous part of (3.12) in the form

$$
\begin{aligned}
& y_{j}=-\mathfrak{g}_{j} V y_{j}+i \mathfrak{g}_{j} {\left[\sum_{m \geq 0} \Omega_{-m} y_{m+j}(x)\right.} \\
&\left.+\sum_{0 \leq l \leq j-j_{0}} \Omega_{l} y_{j-l}(x)+\sum_{l \geq j-j_{0}} \Omega_{l} y_{j-l}(x)\right] \\
&=-\mathfrak{g}_{j} V y_{j}+ i \mathfrak{g}_{j}\left[\sum_{m \geq 0} \Omega_{-m} y_{m+j}(x)+\sum_{0 \leq l \leq j-j_{0}} \Omega_{l} y_{j-l}(x)\right]+E_{j}(x) \\
&=:\left(\mathfrak{T}_{j_{0}} y+E\right)_{j}
\end{aligned}
$$

Since $\left\|\Omega_{j}\right\|_{L^{2}(B)}=O\left(j^{-2}\right)$ and $y \in l^{2}\left(L^{2}(B)\right)$ we see that $\|E\|_{j_{0}}<\infty$. It can be checked that $\mathfrak{T}_{j_{0}}: \mathcal{B}_{j_{0}} \rightarrow \mathcal{B}_{j_{0}}$ is bounded, that $\left\|\mathfrak{T}_{j_{0}}\right\| \rightarrow 0$ as $j_{0} \rightarrow \infty$, and thus eq. (8.26) is contractive if $j_{0}$ is large. The Proposition follows.

Acknowledgments. We are very grateful to K. Yajima for many useful comments and suggestions, including the argument in (7.13). We thank A. Soffer for helpful 


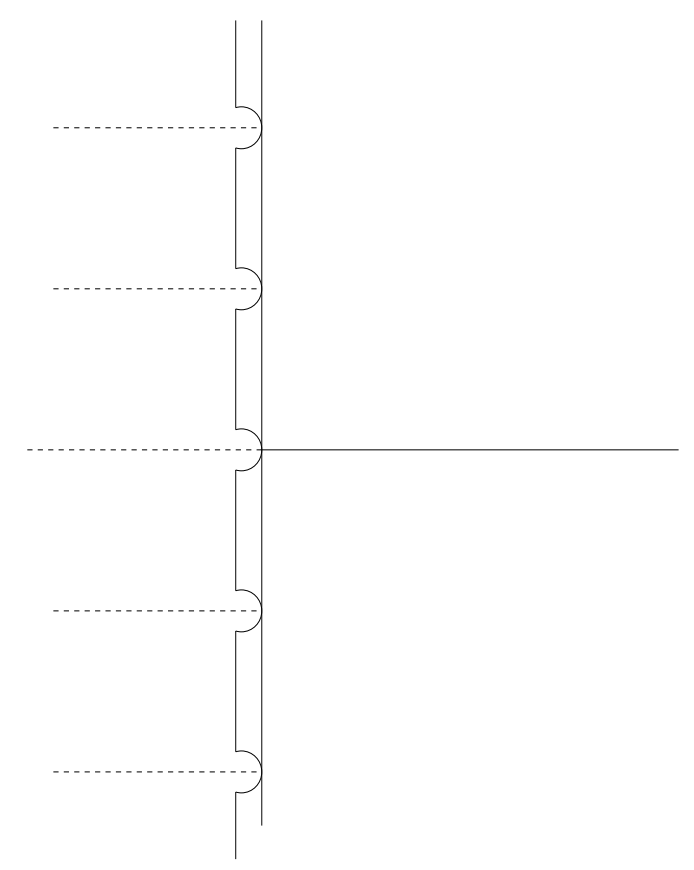

Figure 2. Deformation of the integration contour.

discussions. Work supported by NSF Grants DMS-0100495, DMS-0074924, DMR9813268, and AFOSR Grant F49620-01-1-0154.

\section{REFERENCES}

[1] S Agmon Spectral properties of Schrödinger operators and scattering theory, Ann. Scuola. Norm. Sup. Pisa, Ser. IV 2, pp. 151-218 (1975).

[2] J Belissard, Stability and Instability in Quantum Mechanics, in Trends and Developments in the Eighties (S Albeverio and Ph. Blanchard, ed.) World Scientific, Singapore 1985, pp. $1-106$.

[3] C Bender and S Orszag, Advanced Mathematical Methods for scientists and engineers, McGraw-Hill, 1978, Springer-Verlag 1999.

[4] C Cohen-Tannoudji, J Duport-Roc and G Arynberg, Atom-Photon Interactions, Wiley (1992).

[5] O Costin On Borel summation and Stokes phenomena for rank one nonlinear systems of ODE's Duke Math. J. Vol. 93, No 2: 289-344, 1998

[6] O Costin, R D Costin and J Lebowitz, "Transition to the continuum of a particle in timeperiodic potentials" in Advances in Differential Equations and Mathematical Physics, AMS Contemporary Mathematics series ed. Karpeshina, Stolz, Weikard, and Zeng (2003). 
[7] O Costin, J Lebowitz and A Rokhlenko, Exact Results for the Ionization of a Model Quantum System J. Phys. A: Math. Gen. 33 pp. 1-9 (2000)

[8] O Costin, R D Costin, J Lebowitz and A Rokhlenko, Evolution of a model quantum system under time periodic forcing: conditions for complete ionization Comm. Math. Phys. 221, 1 pp 1-26 (2001).

[9] O Costin, A Rokhlenko and J Lebowitz, On the complete ionization of a periodically perturbed quantum system CRM Proceedings and Lecture Notes 27 pp 51-61 (2001).

[10] O Costin and A Soffer, Resonance Theory for Schrödinger Operators Commun. Math. Phys. 224 (2001).

[11] O Costin, R D Costin, J L Lebowitz (in preparation).

[12] O Costin, R D Costin, Rigorous WKB for discrete schemes with smooth coefficients, SIAM J. Math. Anal. 27, no. 1, 110-134 (1996).

[13] H L Cycon, R G Froese, W Kirsch and B Simon, Schrödinger Operators, Springer-Verlag (1987).

[14] J Écalle, Fonctions Resurgentes, Publications Mathematiques D'Orsay, 1981

[15] J Écalle, in Bifurcations and periodic orbits of vector fields, NATO ASI Series, Vol. 408, 1993

[16] A Galtbayar, A Jensen and K Yajima, Local time-decay of solutions to Schrödinger equations with time-periodic potentials (J. Stat. Phys., to appear).

[17] L Hörmander, Linear partial differential operators, Springer (1963).

[18] J S Howland, Stationary scattering theory for time dependent Hamiltonians. Math. Ann. 207, 315-335 (1974).

[19] H R Jauslin and J L Lebowitz, Spectral and Stability Aspects of Quantum Chaos, Chaos 1, 114-121 (1991)

[20] T Kato, Perturbation Theory for Linear Operators, Springer Verlag (1995).

[21] P D Miller, A Soffer and M I Weinstein, Metastability of Breather Modes of Time Dependent Potentials, Nonlinearity Volume 13 (2000) 507-568.

[22] C Miranda, Partial differential equations of elliptic type, Springer-Verlag (1970).

[23] M Reed and B Simon, Methods of Modern Mathematical Physics (Academic Press, New York, 1972).

[24] A Rokhlenko, O Costin, J L Lebowitz, Decay versus survival of a local state subjected to harmonic forcing: exact results. J. Phys. A: Mathematical and General 35 pp 8943 (2002).

[25] S Saks and A Zygmund, Analytic Functions, Warszawa-Wroclaw (1952).

[26] B Simon, Schrödinger Operators in the Twentieth Century, Jour. Math. Phys. 41, 3523 (2000).

[27] A Soffer and M I Weinstein, Nonautonomous Hamiltonians, Jour. Stat. Phys. 93, 359-391 (1998).

[28] F Treves, Basic linear partial differential equations, Academic Press (1975).

[29] W Wasow, Asymptotic expansions for ordinary differential equations, Interscience Publishers (1968).

[30] K Yajima, Scattering theory for Schrödinger equations with potentials periodic in time, J. Math. Soc. Japan 29 pp 729 (1977)

[31] K Yajima, Existence of solutions of Schrödinger evolution equations, Commun. Math. Phys. 110 pp 415 (1987).

[32] A H Zemanian, Distribution theory and transform analysis, McGraw-Hill New York (1965). 


\section{Welcome to the deleq package!}

This is a short document to demonstrate the use of the deleq package and its commands. It uses deleq version 4.41 (July 7, 1997). deleq was written by Mats Dahlgren (matsd@sssk. se http://www.homenet.se/matsd/). Suggestions for improvements and bug reports are most welcome, see the documentation. deleq is fully compatible with the leqno option and most of the fleqn option.

We start this demonstration by a simple and well-known equation to get the equation number counter going:

$$
\sin ^{2} \alpha+\cos ^{2} \alpha=1
$$

The first example will be to make use of the deqn environment to get a partially numbered equation:

$$
\sin (-\alpha)=-\sin \alpha
$$

This equation has its cosine companion, here written in the ddeqn environment:

$$
\cos (-\alpha)=\cos \alpha
$$

Not so exciting, so far. :-)

In the next example we introduce the deqarr environment for writing equations:

$$
\begin{aligned}
& \sin (\alpha+\beta)=\sin \alpha \cos \beta+\sin \beta \cos \alpha \\
& \sin (\alpha-\beta)=\sin \alpha \cos \beta-\sin \beta \cos \alpha
\end{aligned}
$$

where we also have put in the label Demo1 in the second equation. Next, notice how the environment ddeqar uses the same main equation number as the previous equations:

$$
\begin{aligned}
& \cos (\alpha+\beta)=\cos \alpha \cos \beta-\sin \alpha \sin \beta \\
& \cos (\alpha-\beta)=\cos \alpha \cos \beta+\sin \alpha \sin \beta
\end{aligned}
$$

In the first of these equations, an \arrlabel command with the label Demo2 is included. Now, we will make another eqnarray-like structure, again in the ddeqar environment:

$$
\begin{aligned}
\sin 2 \alpha & =2 \sin \alpha \cos \alpha \\
\cos 2 \alpha & =\cos ^{2} \alpha-\sin ^{2} \alpha
\end{aligned}
$$

or

$$
=2 \cos ^{2} \alpha-1
$$

This example shows the use of \heqno in the first equation, which produces an ordinary equation number. The first equation is also labelled, with the label Demo3. The next equation's number was produced by the command \nydeqno. Also, notice the use of \rem\{or\}, which results in the text "or" without changing the alignment. To illustrate the use of $\backslash$ arrlabel \{Demo2\} above, we here make a reference to it: ... in equations 3 ... which was created by typing ' $\backslash l \operatorname{dots}\{\}$ in equations \ref\{Demo2\} \ldots'.

To step the equation number counter, we want the following equations typeset in ordinary eqnarray environment:

$$
\begin{aligned}
\tan ^{2} \alpha & =\frac{\sin ^{2} \alpha}{\cos ^{2} \alpha} \\
\tan \alpha & =\frac{\sin \alpha}{\cos \alpha}
\end{aligned}
$$


The upper equation was given the label Demo4.

Now one of the "recycling" commands is to be demonstrated. First of all, let's make an ordinary reference to equation 4 and then "recycle" it:

$$
\sin 2 \alpha=2 \sin \alpha \cos \alpha
$$

This was obtained with $\$ \$ \ldots$.. $\$$ with the command \reqno\{Demo3\} at the end. Also partially numbered equations can be recycled with the \reqno\{FO0\} command, as with equation $3 \mathrm{~b}$

$$
\sin (\alpha-\beta)=\sin \alpha \cos \beta-\sin \beta \cos \alpha
$$

(However, the use of \rndeqno\{FOO\} and \rdeqno\{FOO\} will produce strange results with two (different) partial equation numbers if F00 refers to a partially numbered equation.)

The next equation was written with $\$$ \$ . . \$ $\$$ and a \deleqno command at the end:

$$
\tan 2 \alpha=\frac{2 \tan \alpha}{1-\tan ^{2} \alpha}
$$

Notice how the main equation number counter is still the same, despite that we now are outside of the ddeqar environment. Also, notice how the use of \reqno above did not affect the equation number counter. The following equation is also set within $\$ \$ \ldots \$$, but it uses the command \nydeleqno to produce an equation number with a new main number:

$$
\sin ^{2} \alpha=1-\cos ^{2} \alpha
$$

Now we will elaborate a little on \rndeqno\{F00\} and \rdeqno\{F00\}. Let us use equation [6] and see what happens if we use the \rndeqno\{F00\} command:

$$
\tan ^{2} \alpha=\frac{\sin ^{2} \alpha}{\cos ^{2} \alpha}
$$

which we rewrite:

$$
\tan ^{2} \alpha=\frac{\sin ^{2} \alpha}{1-\sin ^{2} \alpha}
$$

with \rdeqno\{Demo4\} at the end. This is great fun, so why not one more:

$$
\tan ^{2} \alpha=\frac{1-\cos ^{2} \alpha}{\cos ^{2} \alpha}
$$

Also, the middle form of equation 6 got a label, Demo5. In the last example, the construct

$$
\text { \ddeqreqno [-\jotbaseline] \{Demo4\} \nonumber }
$$

in a deqrarr environment is used, to obtain fleqn compatibility.

There is still one equation to write. This time we again use the deqarr environment:

where

$$
\cot \alpha=\frac{\cos \alpha}{\sin \alpha}
$$

$$
=\frac{1}{\tan \alpha}
$$

This example also shows the use of the \where command, which is a special case of the \rem command. Now, the interesting thing of referring to the recycled equations is ahead. If one writes \ref $\left\{\right.$ Demo5\}, this is what $\mathrm{LAT}_{\mathrm{E} X} \mathrm{X}$ will return: $\mathrm{b}$. That is not too instructive, since the partial equation numbers are quite common in this document. To make the complete 
reference, use $\backslash \operatorname{ref}\{$ Demo4\} $\backslash \operatorname{ref}\{$ Demo5\}. The reference $[b]$ is much more comprehensible, right? (If you get bad line-breaks at such references, put them in an \mbox\{...\}.)

Now we will show the use of the commands to recycle equation numbers in eqnarray-like structures. These commands are \eqreqno\{FOO\}, \deqreqno\{FOO\}, and \ddeqreqno\{F00\}. We start by repeating equation $3 \mathrm{~b}$ in an deqrarr environment:

$$
\begin{aligned}
\sin (\alpha-\beta) & =\sin \alpha \cos \beta-\sin \beta \cos \alpha \\
\sin 2 \alpha & =2 \sin \alpha \cos \alpha
\end{aligned}
$$

The second equation here shows how the alignment of the equations is preserved. Next, we can use the equation 4 for some variations:

$$
\begin{aligned}
\sin 2 \alpha & =2 \sin \alpha \cos \alpha \\
\sin 4 \alpha & =2 \sin 2 \alpha \cos 2 \alpha \\
& =2\left(2 \sin \alpha \cos \alpha\left(\cos ^{2} \alpha-\sin ^{2} \alpha\right)\right) \\
& =4\left(\sin \alpha \cos \alpha\left(\cos ^{2} \alpha-\sin ^{2} \alpha\right)\right)
\end{aligned}
$$

The features used here are \deqreqno\{Demo3\} on the first line, \ddeqreqno\{Demo3\} on the second line, and \deqreqno[-\jotbaseline] \{Demo3\} on the third. Note how the inclusion of $\left[-\backslash\right.$ jotbaseline] on the third line prevents $\mathrm{LAT}_{\mathrm{E}} \mathrm{X}$ from inserting an extra blank line. The second line ends with ' $\ \backslash$ ', and has thus no equation number at all.

The last part shows how you may use leqreqno and its relatives in deqarr environment:

$$
\begin{aligned}
\sin \alpha & =\sin \alpha \cos 0+\sin 0 \cos \alpha \\
\sin 2 \alpha & =2 \sin \alpha \cos \alpha \\
\sin 3 \alpha & =\sin 2 \alpha \cos \alpha+\sin \alpha \cos 2 \alpha \\
\sin 4 \alpha & =2 \sin 2 \alpha \cos 2 \alpha \\
\sin 5 \alpha & =\sin 3 \alpha \cos 2 \alpha+\sin 2 \alpha \cos 3 \alpha \\
\sin 6 \alpha & =2 \sin 3 \alpha \cos 3 \alpha
\end{aligned}
$$

The interesting part here is an '\eqreqno\{Demo3\}' at the end of the second line and a ' 'ddeqreqno\{Demo3\}' at the end of the fifth line. Notice how the partial equation number counter in unaffected by the enetering of the deqarr environment.

Finally, the $\mathcal{E N D}$ ! If you want your equation numbers left-aligned, just specify the leqno option for the documentclass you are using. It should work! If you have any suggestions, corrections or contributions, please contact me. Enjoy $\mathrm{ITT}_{\mathrm{E} X}$ !

mats $d$. 